\title{
Vitreous fluorophotometry in optic nerve disease
}

\author{
LAURENCE S. BRAUDE, ${ }^{2}$ JOSE G. CUNHA-VAZ,' AND MARCEL FRENKEL' \\ From the 'Department of Ophthalmology, University of Illinois Eye and Ear Infirmary, and the West Side \\ Veterans Administration Hospital, Chicago, USA
}

SUMMARY Posterior vitreous fluorophotometry measurements have been shown to be increased in patients with acute retrobulbar neuritis. We studied 12 patients who had optic neuropathy by posterior vitreous fluorophotometry, with and without correction for blood fluorescein concentration. Both uncorrected and corrected posterior vitreous measurements were increased in patients with inflammatory, obstructive, and infiltrative optic neuropathies. Concurrent values from anterior chamber fluorophotometry were not increased in any of the 8 patients studied.

Inflammation of the optic nerve head includes both clinically visible and inapparent abnormality of the optic nerve. We refer here to optic neuritis as apparent optic nerve head inflammation indicated by clinical and/or fluorescein angiographic abnormality. Retrobulbar neuritis is defined as optic nerve head inflammation not detectable by ophthalmoscopic or fluorescein angiographic abnormality.

It has been shown that posterior vitreous fluorophotometry readings increase in acute attacks of retrobulbar neuritis and return to normal levels on resolution.' Recurrences of retrobulbar neuritis also give increased readings on posterior vitreous fluorophotometry. ${ }^{1}$ The purpose of the present study was to investigate optic neuropathies by vitreous fluorophotometry, and we emphasise the value of this technique when fluorescein angiography gives normal results. Recent modifications have been made in the interpretation of vitreous fluorophotometry readings, by the use of blood fluorescein concentrations. ${ }^{2}$ These were used in the majority of patients studied. We also investigated the value of concurrent fluorophotometric readings in the anterior chamber.

\section{Materials and methods}

Twelve patients with optic nerve disease from the Ophthalmology and Neurology Departments of the University of Illinois and West Side Veterans Administration Hospitals were examined. Both eyes of all patients were examined. Three patients had

Correspondence to Laurence S. Braude. MB, BCh, University of Illinois Hospital Eye and Ear Infirmary, $1855 \mathrm{~W}$ Taylor Street, Chicago, Illinois 60612, USA. retrobulbar neuropathy: cases 1 and 2 had unilateral idiopathic retrobulbar neuritis, and case 3 had ethambutol/isoniazid (toxic) retrobulbar neuropathy (Table 1). Four patients had optic atrophy in one eye: case 4 had multiple sclerosis, cases 5 and 6 had recurrent idiopathic optic neuritis, and case 7 had systemic lupus erythematosus and optic neuritis (Table 1). Cases 4 and 5 had optic neuritis superimposed on optic atrophy. Five patients had clinical and/or angiographic evidence of disc oedema and/or hyperaemia: case 8 had bilateral idiopathic optic neuritis, cases 9 and 10 had unilateral idiopathic optic neuritis, case 11 had unilateral (presumed sarcoid) optic neuritis, and case 12 had bilateral papilloedema secondary to obstructive hydrocephalus (Table 2).

All 12 had a complete eye examination, which included best corrected distance and near vision (at 14 inches $(36 \mathrm{~cm})$ with a Lebensohn chart). The neuroophthalmological examination included pupil assessment (particularly to detect an afferent pupillary defect), motility, nystagmus, colour vision comparison between the eyes (red and green bottle tops and/or Hardy-Rand-Rittler plates), black-andwhite Amsler grid, and Goldmann visual fields of both eyes. Slit-lamp biomicroscopy, applanation tonometry, and dilated fundus examination with scleral depression were performed on all patients. Optic nerve assessment was done by at least 2 observers. Fundus photography, fluorescein angiography, and vitreous fluorophotometry were performed on all the patients at least once.

An injection of $25 \%$ sodium fluorescein was given in a dose of $14 \mathrm{mg} / \mathrm{kg}$ of body weight. Fluorescein angiography was performed on the affected eye (or 


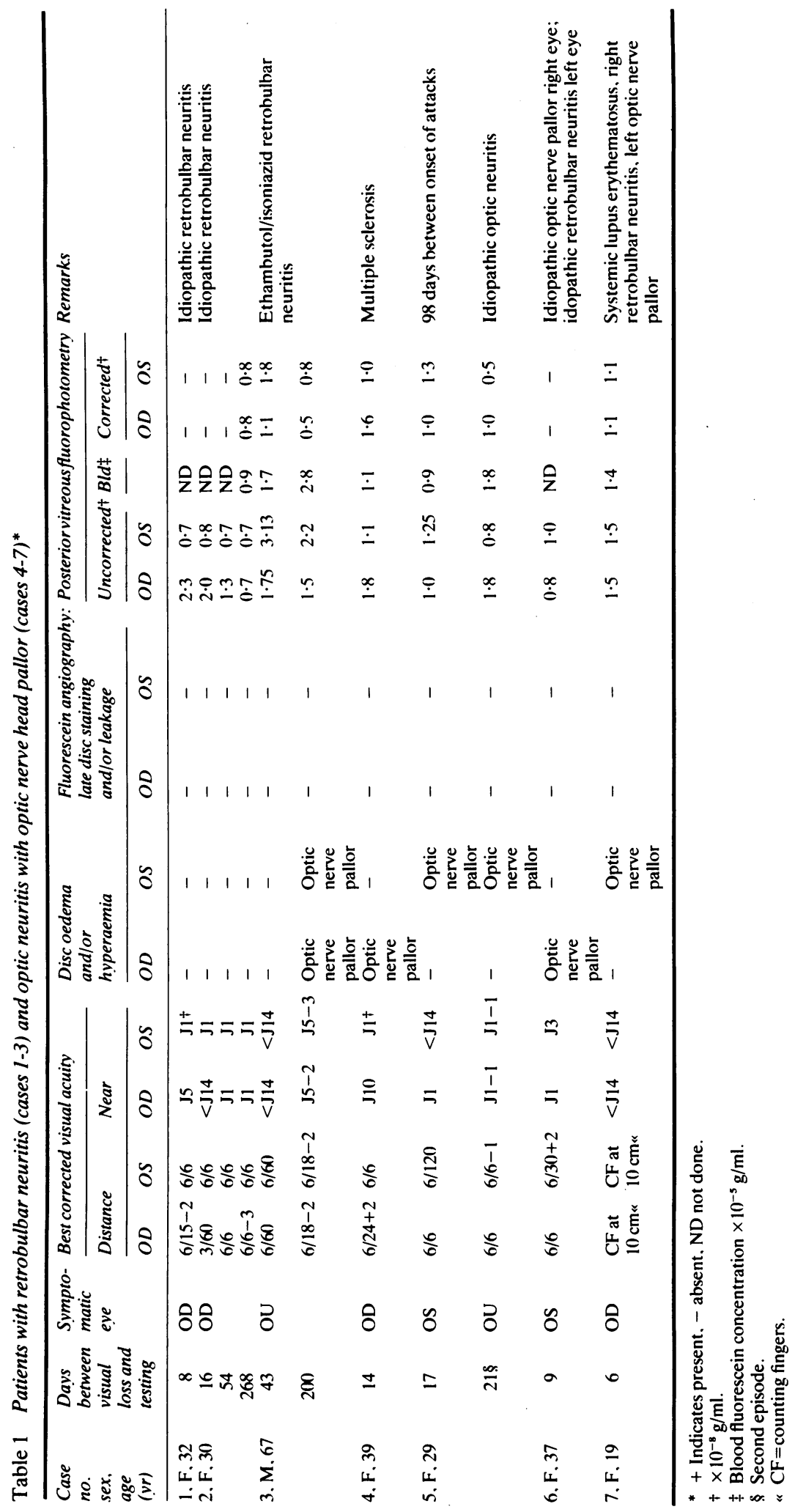




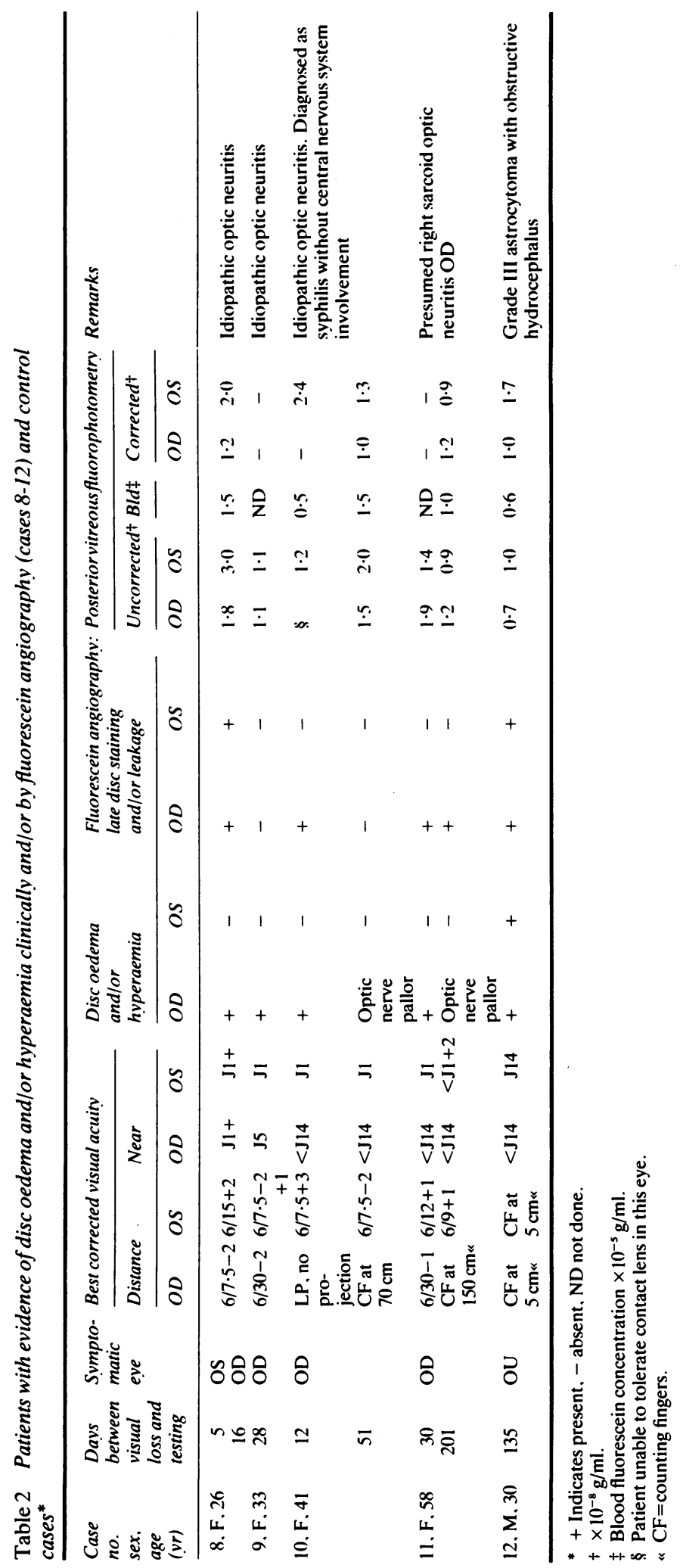


more seriously affected eye if bilateral) immediately on injection, and photographs of both eyes were taken at 10 minutes. Anterior chamber fluorophotometry measurements were made on 8 patients ( 15 eyes) immediately after vitreous fluorophotometry at 1 hour (Table 2). Normal anterior chamber fluorophotometry in our laboratory gives $20 \cdot 9 \pm 12 \cdot 6 \times 10^{-8}$ $\mathrm{g} / \mathrm{ml}$.

Determination of the blood fluorescein concentration was performed in 9 of the 12 patients. Blood from a finger prick was aspirated into a $50 \mu \mathrm{l}$ pipette and then diluted in $2 \mathrm{ml}$ of saline. After centrifugation the fluorescein concentration was measured photometrically in the supernatant diluted plasma. From a study by one of the authors (J. G. C-V.), the posterior vitreous fluorophotometry readings taken $3.5 \mathrm{~mm}$ in front of the retina were corrected for a blood fluorescein concentration of $1 \times 10^{-5} \mathrm{~g} / \mathrm{ml}^{2}{ }^{2}$ We used as a correction factor the ratio between the fluorescein concentration in the blood at 1 hour after injection and $1 \times 10^{-5} \mathrm{~g} / \mathrm{ml}^{2}$ In a recent series of 45 normal volunteers whose background vitreous fluorophotometry readings were corrected in accordance with fluorescein concentrations in the blood a value of $0 \cdot 49 \pm 0 \cdot 17 \times 10^{-8} \mathrm{~g} / \mathrm{ml}($ mean \pm SD) was obtained. In this article posterior vitreous fluorophotometry readings not normalised for blood fluorescein concentration are referred to as uncorrected posterior vitreous fluorophotometry values; normalised readings are referred to as corrected values. Corrected and uncorrected posterior vitreous fluorophotometry readings that were equal to or greater than $1 \times 10^{-8} \mathrm{~g} / \mathrm{ml}$ were considered abnormal. ${ }^{3-5}$ (SI conversion: $\mathrm{g} / \mathrm{l}=\mathrm{g} / \mathrm{ml} \times 10^{3}$.)

All patients had complete neurological and physical examinations (except case 1, which was lost to follow-up). Where indicated, diseases associated with optic neuritis and retrobulbar neuritis were excluded by appropriate investigation.

\section{Results}

\section{RETROBULBAR NEUROPATHY}

The symptoms and signs of retrobulbar neuritis were confirmed with increased posterior vitreous fluorophotometry readings. In cases 1,2 , and 6 these were uncorrected values; in cases 3 and 7 they were corrected readings. Cases 6 and 7 had evidence of optic nerve pallor in the contralateral eye. All cases had idiopathic retrobulbar neuritis except cases 3 and 7 . which were, respectively, toxic retrobulbar neuropathy and systemic lupus erythematosus with right retrobulbar neuritis (Table 1).

OPTIC ATROPHY

Increased corrected posterior vitreous fluorophoto- metry readings confirmed the diagnosis of optic neuritis in 2 patients with optic atrophy in the svmptomatic eye (cases 4 and 5) (Table 1).

\section{DISC OEDEMA AND/OR HYPERAEMIA}

As expected, clinical and/or angiographic evidence of disc oedema or hyperaemia was confirmed, with increased posterior vitreous fluorophotometry readings. In cases 9 and 11 this was demonstrated with increased uncorrected readings on posterior vitreous fluorophotometry. Bilateral confirmation was obtained with increased corrected posterior vitreous fluorophotometry values in cases 8 and 12 .

Asymptomatic fellow eyes had increased uncorrected (cases 9 and 12) and corrected (cases 4, 5, 7. and 10) values on posterior vitreous fluorophotometry. The high incidence of bilaterally increased values on posterior vitreous fluorophotometry in patients with systemic or toxic causes is notable (case 3 , toxic retrobulbar neuropathy; case 7 . systemic lupus erythematosus optic neuritis; and case 11 . presumed sarcoid optic neuritis). Case 10 had a diagnosis of syphilis with a positive serum fluorescent treponemal antibody test and positive serum Reiter protein reaction of 1:4 dilutions. The results from cerebrospinal cytology, protein estimations, and electrophoresis were normal, and the cerebrospinal fluid fluorescein treponemal antibody test was unreactive. Case 11, a 58-year-old black woman, was diagnosed as having presumed sarcoidosis with serum polyclonal gammopathy and serum angiotensin converting enzyme of $40 \mathrm{IU} / \mathrm{I}$ (normal: 10 to $30 \mathrm{IU} / \mathrm{l}){ }^{6}$ cerebrospinal fluid pleocytosis, old right lower lobe granuloma on chest roentgenography, and optic nerve enlargement on computerised tomography (Fig. 1). The serum lysozyme level was $11.4 \mu \mathrm{g} / \mathrm{ml}$ (normal: 2.5 to 12.9 $\mu \mathrm{g} / \mathrm{ml}$ ). The results of a cervical lymph node biopsy were negative in this patient.

The anterior chamber fluorophotometry values at 1 hour after injection in 8 patients ( 15 eyes) were normal (Table 3 ). The mean anterior chamber fluorophotometry values for these patients were $19 \cdot 7 \times 10^{-8} \mathrm{~g} / \mathrm{ml}\left(\mathrm{SD} 6 \cdot 2 \times 10^{-8} \mathrm{~g} / \mathrm{ml}\right.$ ). (SI conversion: $\mathrm{g} / \mathrm{l}=\mathrm{g} / \mathrm{ml} \times 10^{3}$.)

\section{Discussion}

Vitreous fluorophotometry is a sensitive indicator of alteration of the blood-ocular barrier in diabetes and retinitis pigmentosa. ${ }^{27}$ Isolated acute retrobulbar neuritis and acute retrobulbar neuritis associated with multiple sclerosis have been shown to alter the bloodocular barrier reversibly.'

The present study was performed to investigate posterior vitreous fluorophotometry values in 

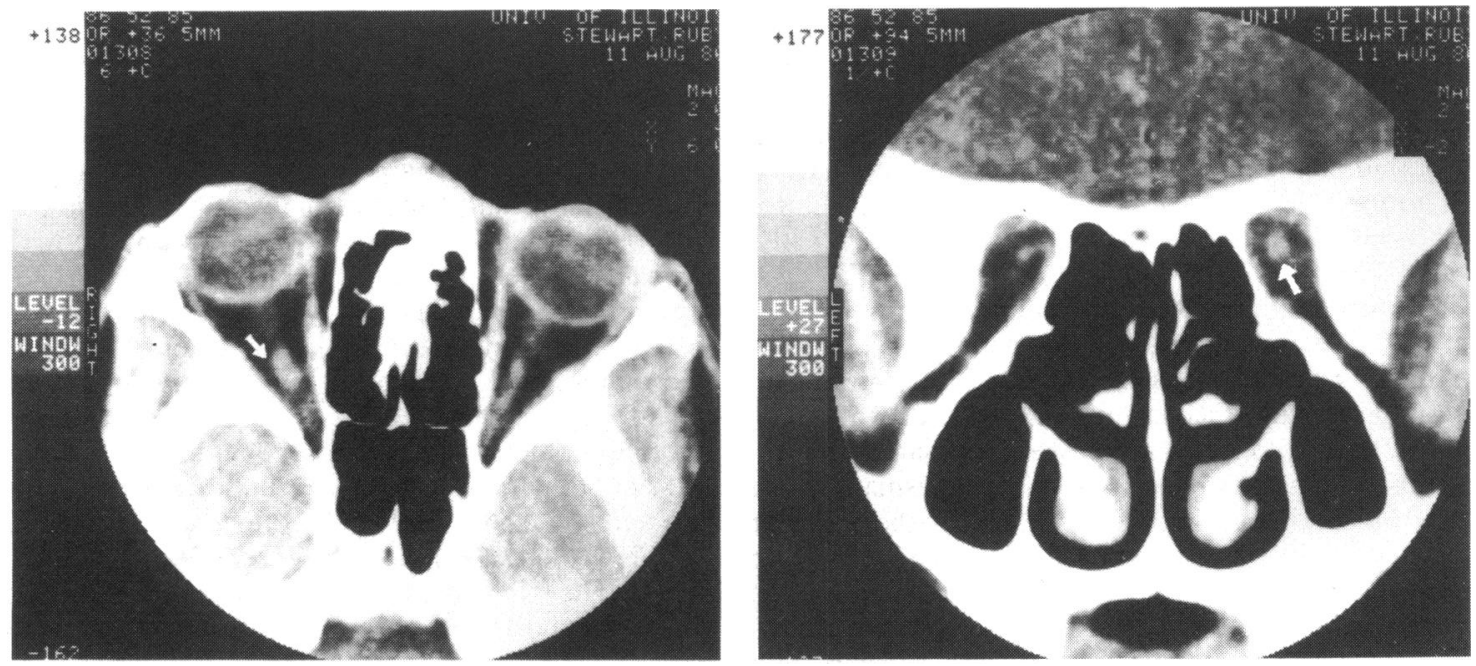

Fig. 1 Case 11 has sarcoid optic neuritis. Note thickening of the posterior half of the right optic nerve (arrows) with apical soft tissue fullness around the optic nerve with contrast infusion on axial computed tomographv (left) and on coronal computed tomography (right).

patients with optic neuropathy with and without correction for variations in blood fluorescein concentration. ${ }^{2}$ Although it is given in a weighted dose. fluorescein has been found to be present in lower levels in the blood of diabetic patients than in normal volunteers. ${ }^{2}$ By normalising the posterior vitreous fluorophotometry value with the blood fluorescein concentration, the corrected posterior vitreous fluorophotometry reading is lowered if the fluorescein concentration in the blood is greater than $1 \times 10^{-5} \mathrm{~g} / \mathrm{ml}$ and increased if the concentration is lower than that level. ${ }^{2}$

RETROBULBAR NEURITIS

Increased corrected posterior vitreous fluoro-

Table 3 Anterior chamber fluorophotometry readings at 1 hour

\begin{tabular}{cclc}
\hline Case & $\begin{array}{l}\text { Davs between } \\
\text { visual loss and } \\
\text { testing }\end{array}$ & \multicolumn{2}{l}{ Anteriorchamber fluorophotometry* } \\
\cline { 3 - 4 } & 268 & Right eve & Left eve \\
\hline 2 & 14 & $11 \cdot 0$ & $9 \cdot 0$ \\
4 & 17 & $29 \cdot 0$ & $22 \cdot 0$ \\
5 & $21^{+}$ & $14 \cdot 5$ & $17 \cdot 0$ \\
& 6 & $30 \cdot 0$ & $28 \cdot 0$ \\
7 & 28 & $14 \cdot 0$ & $14 \cdot 0$ \\
9 & 12 & $20 \cdot 0$ & $19 \cdot 0$ \\
10 & 201 & $\ddagger$ & $25 \cdot 0$ \\
11 & 135 & $23 \cdot 0$ & $22 \cdot 0$ \\
12 & $19 \cdot 0$ & $19 \cdot 0$ \\
\hline
\end{tabular}

$* \times 10^{-5} \mathrm{~g} / \mathrm{ml}$.

+ Second episode.

$¥$ Patient unable to tolerate contact lens in this eye. photometry values in cases 3 and 7 confirm the previous findings of increased uncorrected posterior vitreous fluorophotometry readings in acute retrobulbar neuritis (cases 1,2 , and 6). ${ }^{\prime}$

Case 3 demonstrates retrobulbar neuropathy due to ethambutol/isoniazid toxicity without vitamin $B_{6}$ supplementation. The patient was aphakic $8^{1 / 2}$ years before the onset of visual impairment. Three months before referral the distance visual acuity had been 6/6-1 on the right and 6/7.5-2 on the left. The dosages were $300 \mathrm{mg} /$ day of isoniazid and $1200 \mathrm{mg} /$ day of ethambutol for 13 months until 17 days after the bilaterally decreased visual acuity had been appreciated subjectively. He was referred to us 43 days after subjective visual loss, when distance vision was $6 / 60$ bilaterally. The diagnosis of retrobulbar neuropathy secondary to drug toxicity was confirmed by the bilaterally increased corrected posterior vitreous fluorophotometry values. The patient was started on a regimen of multivitamins and pyridoxine from the day of referral. After 6 months the distance visual acuity had improved to $6 / 18-2$ bilaterally. The corrected posterior vitreous fluorophotometry values at this time had returned to normal.

\section{OPTIC ATROPHY}

Optic nerve pallor indicates previous optic nerve disease and may be associated with little or no disc oedema, depending on the degree of axonal degeneration. Persistent visual evoked response abnormalities may also preclude a diagnosis of acute retrobulbar neuritis in a patient with previous optic 
neuritis, as abnormalities of the visual evoked response can persist for at least 5 years. ${ }^{8}$ Prolongation of latency of a previously increased visual evoked response or decreased amplitude may provide helpful evidence in these cases. Cases 4 and 5 had increased corrected posterior vitreous fluorophotometry values in the symptomatic eyes, which indicated altered permeability of the blood-ocular barrier together with optic atrophy.

\section{DISC OEDEMA AND/OR HYPERAEMIA}

Evidence of disc oedema from ophthalmoscopy or fluorescein angiography or both was confirmed with corrected posterior vitreous fluorophotometry values in cases 8 and 12 . In addition to providing objective confirmation of disc oedema and/or hyperaemia, posterior vitreous fluorophotometry measures leakage and can be helpful in (1) evaluating the progression of disease and response to treatment; (2) differentiating cases that are difficult to differentiate from normal papillary and peripapillary fluorescence on fluorescein angiography; (3) assessing asymptomatic fellow eyes (cases 4, 5, 9, 10, and 11); and (4) assessing asymptomatic fellow eyes in patients with retrobulbar neuritis. ${ }^{1}$

Vitreous fluorophotometry values tend to become normal in approximately 7 weeks in patients with acute retrobulbar neuritis with or without multiple sclerosis. ' Case 2 showed a persistently increased uncorrected value on posterior vitreous fluorophotometry at 54 days. The increased value in this case may relate to persistent symptoms, persistent central Amsler grid distortion, and colour desaturation of the right eye despite $6 / 6$ distance visual acuity. Presumed sarcoid optic neuritis (case 11) is an additional factor that may prolong permeability of the blood-ocular barrier at 6 months and longer following the onset of symptoms. Anterior chamber fluorophotometry, if increased, could be valuable to reinforce the possibility of a systemic cause altering both the blood-aqueous and the blood-retinal barriers. However, no increased anterior chamber fluorophotometry was noted in the 8 patients studied (Table 3).

Sarcoidosis was the presumptive diagnosis in one case. In a recent review $5 \%$ of patients with sarcoidosis were found to have neurological manifestations, and the optic nerve has been shown to be the second most frequently involved cranial nerve. ${ }^{9}$ Computerised tomography has been valuable in detecting optic neuritis and retrobulbar neuritis. ${ }^{10-12}$ One recently reported case of sarcoid retrobulbar optic neuropathy ${ }^{13}$ failed to show any computerised tomographic abnormality. The optic neuritis of case 11 showed thickening of the posterior half of the right optic nerve and inflammatory reaction replacing fatty tissue at the orbital apex on computerised tomography (Fig. 1). Thus the infiltrative ophthalmopathy of a granulomatous disease noted clinically, angiographically, and on computerised tomography (case 11, right eye) was confirmed by vitreous fluorophotometry. The increased vitreous fluorophotometry value in the asymptomatic eye was shown objectively only by a distance visual acuity of $6 / 12+1$. The distance vision subsequently improved one line (Snellen chart) in this asymptomatic eye, with a return to normal of the vitreous fluorophotometry value.

We believe that vitreous fluorophotometry measurements are valuable in diagnosing inflammatory, obstructive, and infiltrative optic neuropathies when conventional fluorescein angiography is normal. Concurrent anterior chamber fluorophotometry readings were not increased in the cases studied, but it seems possible for anterior chamber fluorophotometry readings to be raised without any biomicroscopic disease in the anterior segment when there is a systemic cause of the optic neuropathy, thus indicating a generalised rather than localised aetiology. Another clue to systemic pathology is persistently increased values on vitreous fluorophotometry but persistent symptoms-for example. central scotoma-which may blur the distinction between residual optic nerve damage, recurrence, and continuing systemic disease.

This study was supported by a grant from the Kroc Foundation, Santa Ynez, California, and Core Grant EY01792 from the National Institutes of Health. Bethesda, Maryland.

We thank Ms J. Halik for typing the manuscript.

\section{References}

I Braude LS. Cunha-Vaz JG. Goldberg MF. et al. Diagnosing acute retrobulbar neuritis by vitreous fluorophotometry. $\mathrm{Am} \mathrm{J}$ Ophthalmol 1981; 91: 764-73

2 Cunha-Vaz JG. Wong WP. Kiani R. Kinetic vitreous fluorophotometry in normals and diabetic patients. Read in part before the American Academy of Ophthalmologv annual meeting. Atlanta, 5 November 1981.

3 Cunha-Vaz JG. de Abreu JRF. Campos AJ, et al. Early breakdown of the blood-retinal barrier in diabetes. Br J Ophthalmol 1975; 59: 649-56.

4 Cunha-Vaz JG. Fonseca JR. de Abreau JRF. et al. Detection of early retinal changes in diabetes by vitreous fluorophotometry. Diabetes 1979: 28: 16-9.

5 Cunha-Vaz JG. Goldberg MF. Vygantas C, et al. Early detection of retinal involvement in diabetes by vitreous fluorophotometrv. Ophthalmologv 1979; 86: 264-75.

6 Weinreb RN. Kimura SJ. Uveitis associated with sarcoidosis and angiotensin converting enzyme. Am J Ophthalmol 1980); 89: $180-5$.

7 Gieser DK. Fishman GA. Cunha-Vaz JG. X-linked recessive retinitis pigmentosa and vitreous fluorophotometry: a studv of pigmented heterozygotes. Arch Ophthalmol 1980; 98: 307-10.

8 Halliday AM. McDonald WI, Mushin J. Delayed visual evoked response in optic neuritis. Lancet 1972; i: 982-5. 
9 Delanev P. Clinical review: Neurologic manifestations in sarcoidosis: review of the literature, with a report of 23 cases. Ann Intern Med 1977: 87: 336-45.

10 Trokel SL. Hilal SK. Computed tomography of orbital and ocular tumors. In: Jakobiec FA, ed. Ocular and Adnexal Tumors. Birmingham (USA): Aesculapius. 1978: 257.
11 Osher RH. Tomsak RL. Computed tomographic features in optic neuritis. Am J Ophthalmol 1980; 89: 699-702.

12 Krohel GB. Charles H. Smith RS. Granulomatous optic neuropathv. Arch Ophthalmol 1981: 99: 1053-5.

13 Rush JA. Retrobulbar optic neuropathy in sarcoidosis. Ann Ophthalmol 1980; 12: 390-4. 